\title{
Regularization of Diffusion Tensor Maps Using a Non-Gaussian Markov Random Field Approach
}

\author{
Marcos Martín-Fernández ${ }^{1}$, Carlos Alberola-López ${ }^{1 \star}$, Juan Ruiz-Alzola ${ }^{2}$, and \\ Carl-Fredrik Westin ${ }^{3}$ \\ 1 E. T. S. Ingenieros de Telecomunicación. Universidad de Valladolid \\ 47011 Valladolid (SPAIN) \{marcma, caralb\}@tel.uva.es \\ 2 E. T. S. Ingenieros de Telecomunicación. Universidad de Las Palmas \\ 35017 Las Palmas de Gran Canaria (SPAIN) jruiz@dsc.ulpgc.es \\ 3 Brighman and Women's Hospital Harvard Medical School, Dept. of Radiology \\ 02115 Boston, MA. (USA) westin@bwh.harvard.edu
}

\begin{abstract}
In this paper we propose a novel non-Gaussian MRF for regularization of tensor fields for fiber tract enhancement. Two entities are considered in the model, namely, the linear component of the tensor, i.e., how much line-like the tensor is, and the angle of the eigenvector associated to the largest eigenvalue. A novel, to the best of the author's knowledge, angular density function has been proposed. Closed form expressions of the posterior densities are obtained. Some experiments are also presented for which color-coded images are visually meaningful. Finally, a quantitative measure of regularization is also calculated to validate the achieved results based on an averaged measure of entropy.
\end{abstract}

\section{Introduction}

Diffusion Tensor (DT) Magnetic Resonance Imaging (MRI) is a volumetric imaging modality in which the quantity assigned to each voxel of the volume scanned is not a scalar, but a tensor that describes local water diffusion. Tensors have direct geometric interpretations, and this serves as a basis to characterize local structure in different tissues. The procedure by which tensors are obtained can be consulted elsewhere [9. The result of such a process is, ideally speaking, a $3 \times 3$ symmetric positive-semidefinite (psd) matrix.

Tensors support information of the underlying anisotropy within the data. As a matter of fact, several measures of such anisotropy have been proposed out of tensors to make things easier to interpret; see, for instance, [29]. However, these measures rely of the ideal behavior of the tensors, which may be in some cases far from reality due to some sources of noise that may be present in the imaging process itself. As was pointed out in [7, periodic beats of the cerebro-spinal fluid and partial volume effects may add a non-negligible amount of noise to the data, and the result is that the hypothesis of positive-semidefiniteness may not be valid. Authors are aware of this fact, so some regularization procedures have been proposed in the past [56/79].

\footnotetext{
* To whom correspondence should be addressed.
} 
In this paper we focus on regularization of DT maps using Markov Random Fields (MRFs); other regularization philosophies exist (see, for instance, [8] and [10] and references therein) although they will not be discussed in the paper. About MRFs we are aware of the existence of other Markovian approaches to this problem [6.7], in which the method presented is called by the authors the Spaghetti model. These papers propose an interesting optimization model for data regularization. However, some issues could be a matter of discussion. The authors build their MRF on the basis of a neighborhood system that may change through the optimization process, a fact that is not theoretically correct, though acceptable practical results may be obtained. In addition, the transition model used by the authors does not seem to have a clear probabilistic interpretation, but, in our opinion, only a functional interpretation.

We have recently presented another MRF approach to regularization of DT maps [4, which was based on Gaussian assumptions and which operated separately on each tensor component. Results, as visually assessed, were acceptable and the optimization process guaranteed psd solutions. However, the price to pay was the need for discarding sweeps in the stochastic optimization process.

In this paper we describe an alternative methodology to the one reported in [4] in which, for a 2-D case, the psd condition is forced naturally by the model and, for the 3-D case, the two largest eigenvalues are assured to be positive. The model is built upon a Bayesian philosophy in which the two terms, namely, the prior and the likelihood function, have a clear physical meaning. Closed form expression have been obtained for the posterior, so the resulting model has a solid probabilistic foundation and mathematical elegance. The maximum a posteriori (MAP) estimator is found by means of the simulated annealing algorithm 3 .

\section{Basics on Tensor Processing}

It is well known from matrix theory that a psd tensor, say $\mathbf{A}$, can be described in terms of two matrices, specifically, $\mathbf{A}=\mathbf{Q} \mathbf{\Lambda} \mathbf{Q}^{T}$, where $\mathbf{Q}$ is the eigenvector matrix, with eigenvectors located in every column, and $\boldsymbol{\Lambda}$ is a diagonal matrix with entries equal to the corresponding eigenvalues. Eigenvalues $\lambda_{i}$ are known to be real and $\lambda_{i} \geq 0,(i=\{1,2,3\})$. The eigenvectors are orthonormal, so $\mathbf{Q}^{T} \mathbf{Q}=\mathbf{I}$, with $\mathbf{I}$ the identity matrix.

Some scalar measures have been defined to characterize the tensor behavior. One of them 9] is based on three scalar quantities, namely, the linear, planar and spherical measures. The linear component, which is the focus of attention of this paper, is defined by $c_{l}=\frac{\lambda_{1}-\lambda_{2}}{\lambda_{1}}$, in which an ordering of the eigenvalues is implicit, i.e., $\lambda_{1} \geq \lambda_{2}$. This measure should take on values within the range $[0,1]$ provided that the psd condition is satisfied.

\section{The Model}

Our regularization scheme pursues to highlight fiber tracts for further processing; therefore, we have concentrated on the linear component defined before, 
together with the angle that define the direction of the eigenvector associated to the largest eigenvalue of the tensor (apart from the ambiguity in $\pi$ radians inherent in every eigenvector). For simplicity, the model for the scalar measure will be denoted by "amplitude modeling", and the model for the angle "argument modeling".

As for the amplitude modeling, the model will force smoothness in the solution component according to neighboring voxels, and will select a value that lies within the range $[0,1]$. This assures that the two largest eigenvalues are nonnegative. As for the angle, smoothness in the solution components will also be guaranteed together with the appropriate range of possible values to account for the above mentioned ambiguity in $\pi$ radians.

The model, in its current stage, is two dimensional so only one angle with values within $(-\pi / 2, \pi / 2]$ will be dealt with. However, as it will be obvious shortly, the model extends straightforwardly to three dimensions.

\subsection{Amplitude Modeling}

The prior: The linear component of the tensor will be probabilistically modeled be means of a truncated Gaussian prior. This is to force that values in the prior model lie within the interval $[0,1]$ and, in addition, it provides the model with mathematical tractability. Formally, denoting by $x_{s}$ the linear component of a tensor in a pixel located at site $s$, and denoting by $\delta(s)$ the neighbors of site $s$, the probability density function (pdf) will be

$$
f\left(x_{s} / x_{u}, u \in \delta(s)\right)=\frac{1}{\beta_{s}} \frac{1}{\sigma_{s} \sqrt{2 \pi}} e^{-\frac{\left(x_{s}-A_{s}\right)^{2}}{2 \sigma_{s}^{2}}}
$$

for $0 \leq x_{s} \leq 1$, and zero otherwise. The dependence of site $s$ with its neighbors $\delta(s)$ is through the distribution parameters $A_{s}=A_{s}\left(x_{u}, u \in \delta(s)\right)$ and $\sigma_{s}=\sigma_{s}\left(x_{u}, u \in \delta(s)\right)$. This dependence, however, will not be explicitly stated for simplicity. If erfc stands for the complementary error function, the proportionality factor that assures that the area under this density is unity can be shown to be

$$
\beta_{s}=\frac{1}{2}\left(2-\operatorname{erfc}\left(\frac{1-A_{s}}{\sigma_{s} \sqrt{2}}\right)-\operatorname{erfc}\left(\frac{A_{s}}{\sigma_{s} \sqrt{2}}\right)\right)
$$

This distribution has the mean and variance that follows

$$
\begin{aligned}
& \eta_{s}=A_{s}+\frac{\sigma_{s}}{\sqrt{2 \pi}} \frac{1}{\beta_{s}}\left[e^{-\frac{A_{s}^{2}}{2 \sigma_{s}^{2}}}-e^{-\frac{\left(1-A_{s}\right)^{2}}{2 \sigma_{s}^{2}}}\right] \\
& \varsigma_{s}^{2}=\sigma_{s}^{2}+\frac{\sigma_{s}}{\sqrt{2 \pi}} \frac{1}{\beta_{s}}\left[\left(A_{s}-1\right) e^{-\frac{\left(1-A_{s}\right)^{2}}{2 \sigma_{s}^{2}}}-A_{s} e^{-\frac{A_{s}^{2}}{2 \sigma_{s}^{2}}}\right]-\left(\eta_{s}-A_{s}\right)^{2}
\end{aligned}
$$

Due to the Gaussian-like expressions it can be easily seen that the equation system for the maximum likelihood estimation (MLE) of the parameters of the 
distribution, $A_{s}$ and $\sigma_{s}^{2}$, are the same as equations (3) and (4) with $\eta_{s}$ and $\varsigma_{s}^{2}$ substituted by the sample mean and the sample variance respectively. The solution to these equations is found through a simple and fast iterative method which has been derived by the authors.

The transition model: Even though the eigenvalues of a psd tensor are nonnegative, noise in the acquisition process may cause the tensor not to satisfy this restriction, and, consequently, the linear component is not guaranteed to lie within the interval [0,1] anymore. For instance, if $\lambda_{1}>0$ and $\lambda_{2}<0$ then $c_{l}>1$. In this situation, the closer $\lambda_{1}$ to zero, the closer $c_{l}$ to $\infty$. On the other hand, if both eigenvalues are negative, $c_{l}$ is negative, and, again, the closer $\lambda_{1}$ to zero, the closer $c_{l}$ to $-\infty$.

A provision for this can be easily derived by means of a transition noise model. We have resorted to a simple Gaussian noise model, in which the noise variance has to be inferred from the data. Despite its simplicity, results that will be shown seem satisfactory and, in addition, a closed-form expression for the posterior can be easily calculated.

Denoting by $y_{s}$ the observed tensor linear component at site $s$, the pdf of this variable conditioned to a realization of the prior (and its neighborhood) is a Gaussian pdf, the mean of which equals the realization of the prior, and the variance $\sigma_{n}^{2}$ will be inferred from the data. Formally

$$
f\left(y_{s} / x_{s} ; x_{u}, u \in \delta(s)\right)=\frac{1}{\sigma_{n} \sqrt{2 \pi}} e^{-\frac{\left(y_{s}-x_{s}\right)^{2}}{2 \sigma_{n}^{2}}}
$$

How the parameter $\sigma_{n}^{2}$ is estimated will be described in section 4

The Posterior: As it is well-known, Bayes theorem gives a relation between the three probabilistic entities involved in our modeling scenario, i.e.,

$$
f\left(x_{s} / y_{s} ; x_{u}, u \in \delta(s)\right) \propto f\left(y_{s} / x_{s} ; x_{u}, u \in \delta(s)\right) f\left(x_{s} / x_{u}, u \in \delta(s)\right)
$$

Once again, the fact that equation (1) is a truncated Gaussian and that equation (5) is a pure Gaussian makes the analytical determination of the posterior particularly simple. After some algebra one can arrive at

$$
f\left(x_{s} / y_{s} ; x_{u}, u \in \delta(s)\right)=\frac{1}{\beta_{s / y_{s}}} \frac{1}{\sigma_{s / y_{s}} \sqrt{2 \pi}} e^{-\frac{\left(x-A_{s / y_{s}}\right)^{2}}{2 \sigma_{s / y_{s}}^{2}}}
$$

with $0<x_{s} \leq 1$ and zero otherwise, and

$$
A_{s / y_{s}}=\frac{y_{s} \sigma_{s}^{2}+A_{s} \sigma_{n}^{2}}{\sigma_{s}^{2}+\sigma_{n}^{2}} ; \quad \sigma_{s / y_{s}}^{2}=\frac{\sigma_{s}^{2} \sigma_{n}^{2}}{\sigma_{s}^{2}+\sigma_{n}^{2}}
$$

In order for this function to be a truncated Gaussian the proportionality factor $\beta_{s / y_{s}}$ should be as that expressed in equation (2) with the updated parameters as in equation (8). 


\subsection{Eigenvector Argument Modeling}

The prior: The argument of the eigenvector associated to the largest eigenvalue will be modeled by means of an unreported, to the best of the authors' knowledge, angular distribution, which turns out to be periodic with period $2 \pi$. The pdf can be expressed as

$$
f_{\Theta}\left(\theta_{s} / \theta_{u}, u \in \delta(s)\right)=\frac{1}{2 \pi I_{0}\left(\frac{1}{\lambda_{s}}\right)} e^{\frac{\cos \left(\theta_{s}-\varphi_{s}\right)}{\lambda_{s}}}
$$

for $-\pi<\theta \leq \pi$ and zero otherwise. $I_{0}(x)$ stands for the modified Bessel function of order 0 [1. The dependence of site $s$ with its neighbors $\delta(s)$ is through the distribution parameters $\varphi_{s}=\varphi_{s}\left(\theta_{u}, u \in \delta(s)\right) \in(\pi, \pi]$ and $\lambda_{s}=\lambda_{s}\left(\theta_{u}, u \in\right.$ $\delta(s))>0$. As before, this dependence will not be explicitly stated. Clearly this distribution is well-behaved at the extremes of the interval of allowable values since $f_{\Theta}(-\pi)=f_{\Theta}(\pi)$. This should be so since both arguments are physically the same vector pointing direction.

Due to non-linear and non Gaussian nature of the pdf for variable $\theta_{s}$, the expressions for the MLE of the two parameters involved are not simple data averages. After some algebra one can arrive at

$$
\hat{\varphi}_{M L}=\angle\left(\sum_{i=1}^{N} e^{j \theta_{i}}\right) ; \quad \hat{\lambda}_{M L}=\frac{1}{B^{-1}\left(\frac{1}{N} \sum_{i=1}^{N} \cos \left(\theta_{i}-\hat{\varphi}_{M L}\right)\right)}
$$

with $\theta_{i}, i=\{1, \ldots, N\}$ the observations and $B(x)=\frac{d}{d x} \ln I_{0}(x)$, which is a monotonically growing function, and thus invertible. This function is particularly simple to invert due to well-known approximations of function $I_{0}(x)$ which are valid for most of the domain on which this function is defined [1].

It is obvious that eigenvectors have an ambiguity of $\pi$ radians, i.e., if $\boldsymbol{v}_{1}$ is an eigenvector, so is vector $-\boldsymbol{v}_{1}$. Consequently, the pdf in equation (9) should have an interval range of $\pi$ radians, as opposed to $2 \pi$. This is easily accomplished by defining a new angle, $\vartheta_{s}$, which is related to $\theta_{s}$ by means of the transformation $\vartheta_{s}=\theta_{s} / 2$. Consequently, the prior for the argument will be

$$
f\left(\vartheta_{s}\right)=\frac{f_{\Theta}\left(2 \vartheta_{s}\right)}{\frac{1}{2}}=\frac{1}{\pi I_{0}\left(\frac{1}{\lambda_{s}}\right)} e^{\frac{\cos \left(2 \vartheta_{s}-2 \phi_{s}\right)}{\lambda_{s}}}
$$

with $2 \phi_{s}=\varphi_{s}$. This expression is valid within $-\frac{\pi}{2}<\vartheta_{s} \leq \frac{\pi}{2}$ and zero otherwise.

The transition model: The steering direction of the eigenvector associated to the largest eigenvalue may be affected by noise superimposed in the acquisition process. This can be easily incorporated in the model by means of a transition function of the same type as the prior to connect observations with prior knowledge by means of a noise model. Formally

$$
f\left(\psi_{s} / \vartheta_{s} ; \vartheta_{u}, u \in \delta(s)\right)=\frac{1}{\pi I_{0}\left(\frac{1}{\lambda_{n}}\right)} e^{\frac{\cos \left(2 \psi_{s}-2 \vartheta_{s}\right)}{\lambda_{n}}}
$$


The Posterior: Once again, Bayes theorem allows us to write

$$
f\left(\vartheta_{s} / \psi_{s} ; \vartheta_{u}, u \in \delta(s)\right) \propto f\left(\psi_{s} / \vartheta_{s} ; \vartheta_{u}, u \in \delta(s)\right) f\left(\vartheta_{s} / \vartheta_{u}, u \in \delta(s)\right)
$$

Recalling equations (11) and (12) we can write

$$
f\left(\vartheta_{s} / \psi_{s} ; \vartheta_{u}, u \in \delta(s)\right) \propto e^{\frac{\cos \left(2 \psi_{s}-2 \vartheta_{s}\right)}{\lambda_{n}}+\frac{\cos \left(2 \vartheta_{s}-2 \phi_{s}\right)}{\lambda_{s}}}
$$

Doing some algebra, parallel to the analytical characterization of a complex envelope of a narrow band signal, one can arrive at

$$
f\left(\vartheta_{s} / \psi_{s}, \vartheta_{u}, u \in \delta(s)\right)=\frac{1}{\pi I_{0}\left(\frac{1}{\lambda_{s / \psi_{s}}}\right)} e^{\frac{\cos \left(2 \vartheta_{s}-2 \phi_{s} / \psi_{s}\right)}{\lambda_{s} / \psi_{s}}}
$$

for $-\frac{\pi}{2}<\vartheta_{s} \leq \frac{\pi}{2}$ and zero otherwise, with

$$
\begin{aligned}
& \phi_{s / \psi_{s}}=\frac{1}{2} \angle\left(\lambda_{s} e^{j 2 \psi_{s}}+\lambda_{n} e^{j 2 \phi_{s}}\right) \\
& \lambda_{s / \psi_{s}}=\frac{\lambda_{n} \lambda_{s}}{\sqrt{\lambda_{s}^{2}+\lambda_{n}^{2}+2 \lambda_{s} \lambda_{n} \cos \left(2 \psi_{s}-2 \phi_{s}\right)}}
\end{aligned}
$$

\section{Implementation Details}

In the experiments only one angle has been modeled, i.e., the approach is 2-D. The straightforward model extension to 3-D just needs to model a second angle in the same way as we do here. These two angles would uniquely determine (but for the ambiguity in $\pi$ radians) the direction of the eigenvector in the 3-D space. The noise level $\sigma_{n}^{2}$ for the amplitude is estimated once. This parameter is to be selected within the interval $\sigma_{n}^{2} \in\left[\min _{s} \sigma_{y_{s}}^{2}, \overline{\sigma_{y_{s}}^{2}}\right]$, where the latter expresses the arithmetic mean of the values $\sigma_{y_{s}}^{2}$ in every site of the observed field. $\sigma_{y_{s}}^{2}$ is the unbiased sample variance calculated with $N=20$ neighbors of site $s$.

A similar procedure has been used for parameter $\lambda_{n}$ of the transition model for the argument. In this case, $\lambda_{n} \in\left[\min _{s} \lambda_{y_{s}}, \overline{\lambda_{y_{s}}}\right]$, with $\lambda_{y_{s}}$ the MLE of this parameter (equation (10) ) using $N=20$ neighbors of site $s$.

For the optimization process to find the MAP solution of the field we have used the simulated annealing procedure [3] with a partially parallel visit schedule and a logarithmic cooling scheme. The number of sweeps has been, in all the experiments, 15. Subsequent estimations of the posterior parameters (as the optimization process evolves) has been done with 12 neighbors, excluding the pixel under analysis.

In order to quantify the degree of regularization we have calculated a measure of cross-entropy, as follows:

$$
R=-\sum_{m=1}^{N_{R c_{l}}} \sum_{n=1}^{N_{\vartheta}} p_{m n} \log _{2} p_{m n}
$$


with $N_{R c_{l}}$ the number of discrete values considered for the ratio of linear components between two neighboring sites, and $N_{\vartheta}$ the number of discrete values considered for the difference of the arguments in these two sites. The former has been forced to lie within the interval $(0,1)$ by using the largest of the two linear components in the denominator. The angle difference is unwrapped so it lies within $(0, \pi)$. The number of neighbors used to calculate this $20-12=8$ since we have used the nearest neighbors in the 20 -site neighborhood that have not been used in the optimization process referred to above.

\section{Results}

Figure 1 shows both the original (a) and the regularized field (b) with the parameters $\sigma_{n}^{2}$ and $\lambda_{n}$ selected in the mid point of the respective intervals described above. The images are a color-coded composition in which every pixel (i.e. every field site) has an RGB coordinate, with $R=c_{l} \cdot R\left(\vartheta_{s}\right), G=c_{l} \cdot G\left(\vartheta_{s}\right)$ and $B=c_{l} \cdot B\left(\vartheta_{s}\right)$. The $R(\cdot), G(\cdot)$ and $B(\cdot)$ functions are periodic with period $\pi$. Consequently every argument has a color associated, and the darker the pixel, the closer to zero the linear component of the tensor in that site.

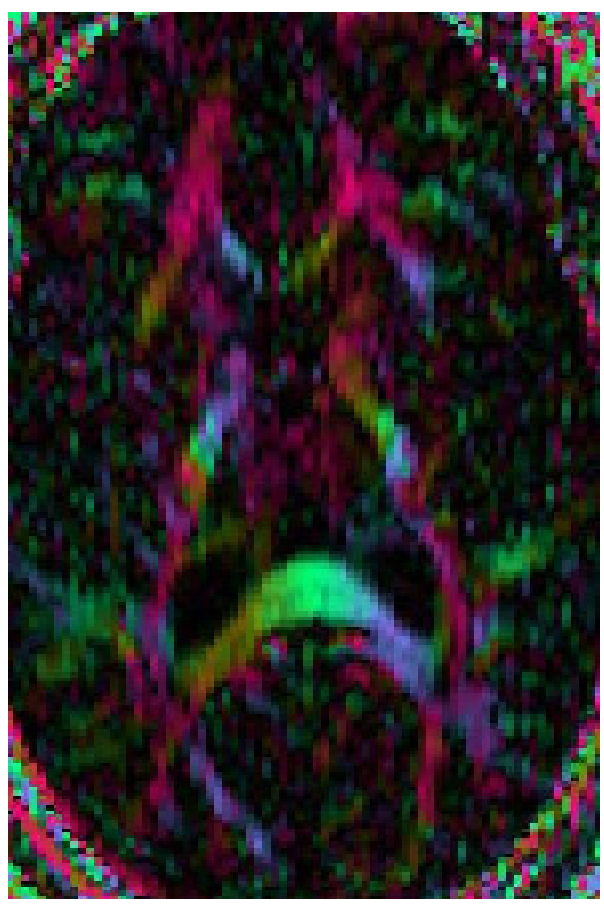

(a)

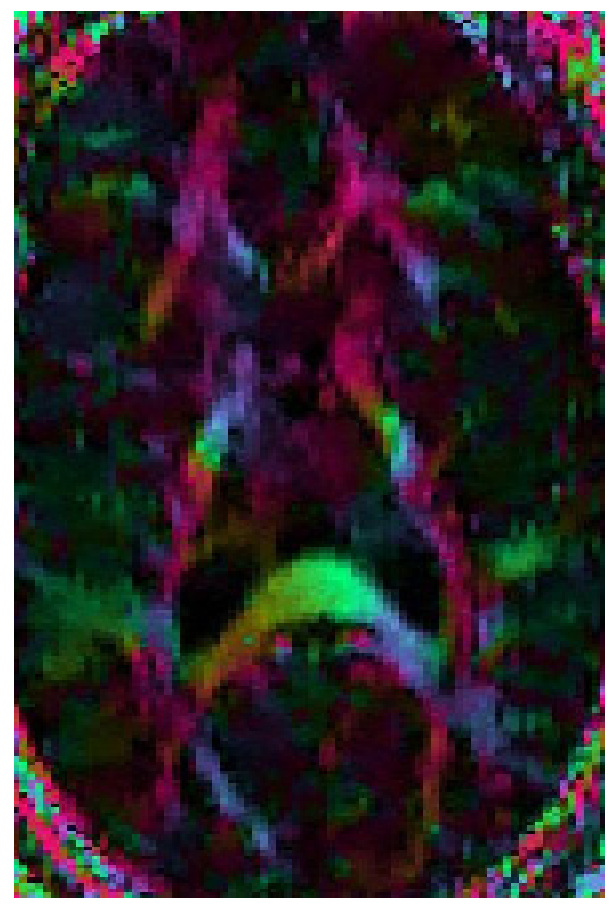

(b)

Fig. 1. (a) Color-coded image in original field. (b) Regularized field. 
The values of the entropies, as defined above, are 5.8038 for the original image and 5.1864 for the processed image. Visual assessment of the regularized fields matches numerical results.

\section{Conclusions}

In this paper we have described a novel probabilistic Bayesian model for the regularization of DT maps. We have only taken into account the linear tensor component together with the direction of the eigenvector associated to the largest eigenvalue, an information which should be sufficient for a tractography application; we are aware of the lack of stability in DT-MRI tractography based only on this direction. However, the linear component adds information about how much the argument of the eigenvector should be trusted in such an application. A direct extension of the method for the three dimensional case, as pointed out in the paper, is fairly straightforward. However, taking the other components (planar and spherical) into account is a matter of further research.

Acknowledgments. The authors acknowledge the Comisión Interministerial de Ciencia y Tecnología for research grant TIC2001-3808-C02, NIH grant P41RR13218 and CIMIT.

\section{References}

1. M. Abramowitz, I. A. Stegun, Handbook of Mathematical Functions with Formulas, Graphs, and Mathematical Tables, Dover, New York, 1972.

2. P. Basser, C. Pierpaoli, Microstructural and Physiological Features of Tissues Elucidated by Quantitative-Diffusion-Tensor MRI, Journal of Magnetic Resonance, Ser. B, Vol. 111, No. 3, June 1996, pp. 209-219.

3. S. Geman, D. Geman, Stochastic Relaxation, Gibbs Distributions and the Bayesian Restoration of Images, IEEE Trans. on PAMI, Vol. 6, No. 6, Nov. 1984, pp. 721741.

4. M. Martín-Fernández, R. San José Estépar, C. F. Westin, C. Alberola-López, A Novel Gauss-Markov Random Field Approach for Regularization of Diffusion Tensor Maps, Proc. of the NeuroImaging Workshop, Eurocast 2003, Las Palmas de Gran Canaria, Feb. 2003, pp. 29-32.

5. G. J. M. Parker, J. A. Schnabel, M. R. Symms, D. J. Werring, G. J. Barker, Nonlinear Smoothing for Reduction of Systematic and Random Errors in Diffusion Tensor Imaging, Journal of Magnetic Resonance Imaging, Vol. 11, No. 6, 2000, pp. 702-710.

6. C. Poupon, J. F. Mangin, V. Frouin, J. Regis, F. Poupon, M. Pachot-Clouard, D. Le Bihan, I. Bloch, Regularization of MR Diffusion Tensor Maps for Tracking Brain White Matter Bundles, in Lecture Notes in Computer Science, W. M. Wells, A. Colchester, S. Delp, Eds., Vol. 1946, Oct. 1998, pp. 489-498.

7. C. Poupon, C. A. Clark, V. Frouin, J. Regis, D. Le Bihan, I. Bloch, J. F. Mangin, Regularization Diffusion-Based Direction Maps for the Tracking of Brain White Matter Fascicles, NeuroImage, Vol. 12, 2000, pp. 184-195. 
8. D. Tschumperlé, R. Deriche, DT-MRI Images: Estimation, Regularization and Application, Proc. of the NeuroImaging Workshop, Eurocast 2003, Las Palmas de Gran Canaria, Feb. 2003, pp. 46-47.

9. C. F. Westin, S. E. Maier, H. Mamata, A. Nabavi, F. A. Jolesz, R. Kikinis, Processing and Visualization for Diffusion Tensor MRI, Medical Image Analysis, Vol. 6, No. 2, June 2002. pp. 93-108.

10. C. F. Westin, H. Knuttson, Tensor Field Regularization using Normalized Convolution, Proc. of the NeuroImaging Workshop, Eurocast 2003, Las Palmas de Gran Canaria, Feb. 2003, pp. 67-70. 